\title{
Die Verwendung vasoaktiver Substanzen in der Diagnostik und Therapie der Erektilen Dysfunktion - Rechtliche Aspekte*
}

\author{
I. Schroeder-Printzen ${ }^{1}$, J. Göben ${ }^{2}$, W. Weidner ${ }^{1}$, R.-H. Ringert ${ }^{1}$ \\ ${ }^{1}$ Klinik und Poliklinik für Urologie (Direktor: Prof. Dr. med. R.-H. Ringert) \\ ${ }^{2}$ Abteilung für Arzt- und Arzneimittelrecht der Georg-August-Universität Göttingen (Leiter: Prof. Dr.jur. E. Deutsch)
}

\section{Zusammenfassung}

Zur Diagnostik und Therapie der erektilen Dysfunktion (E.D.) werden vasoaktive Substanzen wie Papaverin, ein Gemisch von Papaverin/Phentolamin und Prostaglandin $\mathrm{E}_{1}\left(\mathrm{PGE}_{1}\right)$ verwendet. Das Papaverin/ Phentolamin-Gemisch fällt als Rezepturarzneimittel unter die Ausnahmeregelung des \$21 I Nr. 1 AMG und bedarf deshalb keiner Zulassung durch das Bundesgesundheitsamt (BGA). Papaverin und $\mathrm{PGE}_{1}$ sind Fertigarzneimittel im Sinne der $\$ \$ 2,4$ Arzneimittelgesetz (AMG) und sind vom BGA für andere Indikationen zugelassen. Eine Zulassung für die Diagnostik und Therapie der E.D. besteht zur Zeit nicht. Trotzdem dürfen diese Substanzen im Rahmen einer klinischen Prüfung oder als Heilversuch eingesetzt werden. Die klinische Prüfung nach den $\$ \$ 40,41$ AMG ist strengen Voraussetzungen unterworfen. Der Heilversuch im Rahmen der ärztlichen Therapiefreiheit ist eine weitere Möglichkeit, diese Substanzen einzusetzen. Er setzt dabei eine umfangreiche Aufklärung über den Heilversuchscharakter, mögliche Nebenwirkungen und die alternativen Therapiemöglichkeiten voraus. Weiterhin sind die Kontraindikationen $\mathrm{zu}$ beachten.

\section{Vasoactive Drugs for Diagnosis and Treatment of Erectile Dysfunction - Legal Aspects}

For many years vasoactive drugs such as papaverine, papaverine/phentolamine and prostaglandin $\mathrm{E}_{1}\left(\mathrm{PGE}_{1}\right)$ have been used in the diagnosis and treatment of erectile impotence. As defined in $\$ \$ 2,4$ of the German law governing the manufacture and prescription of drugs (AMG) papaverine and $\mathrm{PGE}_{1}$ are prefabricated drugs. The papaverine/phentolamine mixture is a dispensing drug and therefore qualifies for eyemption under \$21 I Nr. 1 AMG. Under special circumstances it does not need to licensed by the German BGA (German aequivalent of the FDA). Although the monosubstances papaverine and $\mathrm{PGE}_{1}$ are licensed by the $\mathrm{BGA}$ for other medical indications, there is no license for using these drugs in the diagnosis and treatment of erectile impotence. This means, that these drugs are only allowed to be used in a clinical experiment or for a healing trial. However, the conditions for the clinical experiment, as laid down in $\$ \$ 40,41 \mathrm{AMG}$, are very strict. A healing trial, conducted under the terms of the doctor's freedom of choice regarding treatment, represents another possibility to use these drugs. Yet for such a trial it is necessary to provide extensive information about the specific nature of the treatment, about the possible side effects and about all alternative forms of treatment. Furthermore, special attention has to be paid to the contraindications.

\section{Key words}

Intracavernosal self-injection therapy Legal viewpoint - Papaverin - Papaverin/Phentolamin Prostaglandin $\mathrm{E}_{1}$

\section{Einleitung}

Seit den ersten Untersuchungen von Virag (23) und Brindley (1) bilden die intrakavernösen Injektionen von vasoaktiven Substanzen einen Eckpfeiler der Diagnostik und Therapie der erektilen Dysfunktion (4).
Weltweit haben sich dabei Papaverin, das Gemisch von Papaverin/Phentolamin und Prostaglandin $\mathrm{E}_{1} \quad \mathrm{PGE}_{1}$ ) für den klinischen Gebrauch durchgesetzt (Übersichten bei 4,26). Weitere Substanzen wie Vasoaktive Intestinal Polypeptide (14), Calcitonin-Gene-Related Peptide (20), Linsidomin Chlorhydrat (SIN-1) (21) sind bis-
Akt. Urol. 23 (1992) 248-251

(c) Georg Thieme Verlag Stuttgart . New York

\footnotetext{
* Auszüge wurden auf dem Norddeutschen Urologenkongreß am 1. Juni 1991 in Hannover vorgestellt.
} 
her Gegenstand klinischer Forschung und werden deswegen nicht weiter behandelt.

\section{Verwendete Substanzen sind Arzneimittel}

Die Einzelsubstanzen Papaverin und $\mathrm{PGE}_{1}$ bzw. das Papaverin/Phentolamin-Gemisch sind Stoffe, die dazu bestimmt sind, im oder am Menschen Krankheiten, Leiden, Körperschäden oder krankhafte Beschwerden zu heilen, zu lindern oder zu erkennen. Sie erfüllen somit die Voraussetzungen des \$2 I Arzneimittelgesetz (AMG) (22) und sind deshalb als Arzneimittel anzusehen. Dabei erfüllen Papaverin und PGE 1 die Voraussetzungen eines Fertigarzneimittels im Sinne des \$4 I AMG, da sie im voraus in großen Mengen gefertigt und zur Abgabe an den Verbraucher abgepackt und abgefüllt werden.

Dagegen wird das Papaverin/Phentolamin-Gemisch nur für einen bestimmten Patienten hergestellt, es ist somit nach §4 I AMG (15) ein Rezepturarzneimittel und kein Fertigarzneimittel. Nach $\$ 21$ II Nr. 1 bedürfen Rezepturarzneimittel unter bestimmten Voraussetzungen (z.B. Chargengröße $<100$ Packungen, Herstellung und Abgabe in derselben Apotheke) keiner Zulassung durch das Bundesgesundheitsamt (BGA) $(15,22)$. Da bei der Herstellung des Gemisches auch Papaverin verwendet wird, können Papaverin-typische Komplikationen auftreten und es sind daher auch dieselben Kontraindikationen zu beachten. Deshalb sollten unserer Ansicht nach auch bei der Verwendung des Papaverin/Phentolamin-Gemisches als Rezepturarzneimittel dieselben Maßstäbe angelegt werden, wie sie im folgenden für Papaverin und $\mathrm{PGE}_{1}$ dargestellt werden.

\section{Rechtliche Grundlagen für einen indikationsfremden Arzneimittelgebrauch}

Nach §21 I AMG dürfen Fertigarzneimittel nur in den Verkehr gebracht werden, wenn sie zugelassen sind. Papaverin und $\mathrm{PGE}_{1}$ sind vom BGA für bestimmte Indikationen (z. B. arterielle Verschlußkrankheit, Migräne, Menièresches Syndrom) zugelassen. Eine Zulassung für die Diagnostik und Therapie der E. D. besteht nicht. Daraus ergibt sich die Frage, ob sie trotzdem eingesetzt werden dürfen. Prinzipiell ist dieses möglich. Es ist dabei zwischen zwei grundsätzlich verschiedenen Möglichkeiten zu unterscheiden:

1. der klinischen Prüfung eines Medikamentes $(\$ \S 40,41$ AMG im Rahmen der Ausnahmeregelung des \$21 II Nr. $2 \mathrm{AMG}$

2. dem Heilversuch im Rahmen der ärztlichen Therapiefreiheit.

\section{Klinische Prüfung im Rahmen der Ausnahmeregelung des $\S 21$ II Nr. 2 AMG}

Diese Vorschrift befreit Substanzen, die bei einer klinischen Prüfung verwendet werden, von der $\mathrm{Zu}$ lassungspflicht. Dabei ist zwischen der klinischen Prüfung als Experiment ( $\$ 40$ AMG) und der klinischen Prüfung als Heilversuch (\$41 AMG) zu unterscheiden.

\section{Klinische Prüfung nach $\$ 40$ AMG}

Der $\$ 40$ AMG nennt die Voraussetzungen, unter denen eine klinische Prüfung als Experiment möglich ist. Die möglichen Risiken für die Versuchsperson im Rahmen einer eng auszulegenden Risiko-Nutzen-Abwägung müssen gemessen an der voraussichtlichen Bedeutung des Arzneimittels vertretbar sein. Weiterhin sind vorherige pharmakologisch-toxikologische Prüfungen unabdingbar. Die Ergebnisse müssen beim BGA hinterlegt werden. Ein Prüfplan, der dem Stand der derzeit gültigen wissenschaftlichen Erkenntnisse entspricht, ist Voraussetzung.

Der Proband muß voll geschäftsfähig und in der Lage sein, die Tragweite der klinischen Prüfung einzusehen. Es hat eine umfassende Aufklärung stattzufinden. Die Einwilligung hat schriftlich zu erfolgen. Weiterhin muß eine Probandenversicherung abgeschlossen werden. Der die klinische Prüfung leitende Arzt muß eine zweijährige Tätigkeit in der klinischen Prüfung von Arzneimitteln nachweisen.

\section{Klinische Prüfung nach $\$ 41$ AMG}

Bei der klinischen Prüfung als kontrollierter Heilversuch nach $\$ 41$ AMG stellt der Gesetzgeber darauf $\mathrm{ab}$, daß dieses Arzneimittel nach den Erkenntnissen der medizinischen Wissenschaft geeignet ist, das Leben eines einzelnen Kranken zu retten, seine Gesundheit wiederherzustellen oder sein Leiden zu erleichtern. Im Gegensatz zum Humanexperiment steht hier nicht die Erweiterung des medizinischen Erkenntnisstandes, sondern der individuelle Nutzen für den Patienten im Vordergrund. Im Gegensatz zu $\$ 40$ AMG dürfen auch nicht vollgeschäftsfähige Personen mit Einwilligung ihres gesetzlichen Vertreters an dieser Form der klinischen Prüfung teilnehmen. Die bereits zu $\$ 40$ AMG genannten anderen Voraussetzungen gelten auch hier.

\section{Heilversuch im Rahmen der ärztlichen Therapiefreiheit}

Unabhängig von den Möglichkeiten die das AMG bietet, hat jeder Arzt im Rahmen seiner Therapiefreiheit die Möglichkeit, ein Medikament außerhalb seines vom BGA zugelassenen Indikationsbereiches einzusetzen $(3,6)$. Gelegentlich ist der allgemeine Stand der medizinischen Erkenntnisse aktueller als das Zulassungsverfahren. Trotzdem unterliegt die Anwendung eines Medikamentes außerhalb seiner zugelassenen Anwendungsgebiete rechtlichen Grenzen (3). Die Rechtsprechung sieht diese als überschritten an, wenn der Einsatz medizinisch nicht geboten ist (10). Medizinische Gebotenheit liegt aber jedenfalls dann vor, wenn das Arzneimittel in seiner beabsichtigten Anwendung medizinisch-wissenschaftlich erprobt und in seinen Nebenwirkungen ausreichend bekannt ist. In Einzelfällen, etwa bei lebensbedrohlichen Erkrankungen, kann sich die Möglichkeit des Arztes zum bestimmungswidrigen Arzneimitteleinsatz sogar zur Pflicht dieses Medikament einzusetzen verdichten (10). 
Tab. 1 Wichtige Kontraindikationen bei der systemischen Gabe von Papaverin, Papaverin/Phentolamin und PGE1.

kardiale: unbehandelte KHK und Herzinsuffizienz, schwere Herzrhythmusstörung, Herzinfarkt $<6$ Monate

pulmonale: Lungenödem, Pneumonie, schwere obstruktive Ventilationsstörung

zerebrale: Krampfanfallsleiden, erhöhter Hirndruck

sonstige: Engwinkelglaukom, Gerinnungs- oder Leberfunktionsstörungen, Obstipation

Diese Pflicht ist sicher bei der Behandlung der E.D. auszuschließen.

Für den Einsatz vasoaktiver Substanzen in der Diagnostik und Therapie der E. D. müssen folgende Voraussetzungen erfüllt sein, wenn ein Heilversuch erfolgen soll:

1. Wirksamkeit der Substanz bei gleichzeitig geringer Nebenwirkungsrate,

2. Beachtung der Kontraindikationen,

3. umfassende Aufklärung des Patienten,

4. Einwilligung des Patienten.

\section{Wirksamkeit}

Die Wirksamkeit von Papaverin, $\mathrm{PGE}_{1}$ und des Papaverin/Phentolamin-Gemisches ist in der Diagnostik und Therapie der E.D. weitgehend unumstritten (4, 26). In der Therapie beschränkt sich die Indikation im wesentlichen auf neurogene und vaskuläre Störungen (4).

\section{Kontraindikationen}

Die in Tab. 1 aufgeführten Kontraindikationen sind vom BGA für eine systemische oder intraarterielle Gabe vorgeschrieben worden. Da weder Papaverin noch $\mathrm{PGE}_{1}$ eine Zulassung für die intrakavernöse Injektion besitzen, existiert keine spezielle Liste von Kontraindikationen für diese Applikationsart. Aus forensischen Gründen scheint es uns zur Zeit trotzdem sinnvoll, sich zunächst an diesen Kontraindikationen zu orientieren. Die Nichtbeachtung von Kontraindikationen führt im Falle eines Prozesses zu einer Beweisbelastung des Arztes bei der Frage der Einhaltung der objektiv erforderlichen Sorgfalt (2). Dieser Beweis ist häufig schwer zu führen.

\section{Aufklärung}

Der ausführlichen, über das Maß des Normalen hinausgehenden, Aufklärung kommt bei einem Heilversuch mit einem Medikament, das für diese Indikation nicht zugelassen ist, eine ganz erhebliche Bedeutung $\mathrm{zu}(3,6)$.

Es muß darauf hingewiesen werden, da $\beta$ es sich um eine Behandlung mit einem Medikament handelt, das für die Indikation E. D. vom BGA keine Zulassung besitzt. Gleichzeitig sind mögliche alternative Therapien (9) mit ihren Erfolgsaussichten und Risiken ausführlich zu erläutern. Dabei ist die Aufklärung individuell auf den
Tab. 2 Durchschnittswerte für das Auftreten von Priapismen in der Diagnostik und Therapie der E.D., differenziert nach Substanzen (zusammengestellt aus 2).

\begin{tabular}{lll}
\hline & Diagnostik & Therapie \\
\hline Papaverin & $9,5 \%$ & $0,4 \%$ \\
Papaverin/ & $5,3 \%$ & $0,4 \%$ \\
Phentolamin & & \\
PGE $_{1}$ & $2,4 \%$ & $0,1 \%$ \\
\hline
\end{tabular}

Patienten abzustimmen. Weiterhin ist über mögliche Nebenwirkungen bzw. Komplikationen ausführlich aufzuklären.

\section{Aufklärungsbedürftige Komplikationen und Nebenwirkungen}

Als häufiger auftretende Komplikationen sollten der Injektions- und Erektionsschmerz genannt werden. Dabei ist zwischen Papaverin und $\mathrm{PGE}_{1}$ zu unterscheiden, gerade bei letzterem Medikament kommt es in ca. $17 \%$ zu diesen Nebenwirkungen, allerdings schwanken die Angaben in der Literatur zwischen 0-59\% (4).

Auf das Auftreten eines Priapismus (Erektion $>6$ Stunden) muß ebenfalls aufgeklärt werden. Dabei ist zwischen dem Auftreten in der Diagnostik und in der Therapie zu differenzieren. Die in Tab. 2 aufgeführten Zahlen sind Durchschnittswerte, die aus einer Vielzahl von Literaturstellen durch Jünemann et al. (4) gewonnen wurden. Es ist ausdrücklich darauf hinzuweisen, daß der Patient innerhalb von 5-6 Stunden einen Urologen aufsuchen muß, da sonst zumindest beim Low Flow Priapismus mit beginnenden irreversiblen Schäden gerechnet werden muß, auch wenn zur Zeit der Zeitpunkt des Schädigungsbeginnes noch nicht exakt definiert ist und individuell unterschiedlich zu sein scheint $(8,12)$.

Hämatome, Unterblutungen oder penile Mißempfindungen (Auftreten in ca. 2-10\%) sind eher auf falsche Injektionstechnik als auf die verwendeten Substanzen zurückzuführen $(4,26)$, dennoch gehören sie in das Aufklärungsgespräch.

Die Cavernitis als schwerste Form der penilen Infektion (16) wird bei der Schwellkörperautoinjektionstherapie (SKAT) mit 0,5\% angegeben (17). Trotz ihres relativ seltenen Auftretens sollte sie erwähnt werden.

Bei den systemischen Nebenwirkungen sind mögliche Kreislaufreaktionen (Schwindel, Synkopen) und Leberfunktionsstörungen zu erwähnen. Zu Kreislaufreaktionen kann es bei höheren Papaverindosen und gleichzeitig bestehendem venösen Leakage kommen (25). Für $\mathrm{PGE}_{1}$ sind Beeinträchtigungen des Kreislaufsystems ebenfalls denkbar, obwohl es wesentlich schneller in der Lunge metabolisiert wird (19).

Erhöhungen der Leberwerte bei Verwendung von Papaverin sind in der Literatur beschrieben (4, 26). Bei den meisten dieser Patienten war gleichzeitig eine 
Alkoholanamnese bekannt, so daß Erhöhungen der Leberenzyme der Papaveringabe nicht eindeutig zuzuordnen waren $(5,7)$. In den wenigen anderen Fällen wurde SKAT abgebrochen (11). Obwohl Veränderungen der Transaminasen selten sind, sollten sie vor und während der Therapie kontrolliert werden. Bei einem Ansteigen ist eine Therapieunterbrechung angebracht.

In der Langzeittherapie bedarf die Entwicklung von penilen Knoten an der Injektionsstelle einer gesonderten Erwähnung. Dabei kann es sich um subkutane, in der Tunica albuginea liegende oder intrakavernöse Knoten handeln (24). Als Ursachen kommen das lokale Injektionstrauma und die Substanzen selbst in Frage, wobei für Papaverin der saure PH als gewebetoxisch diskutiert wird $(4,26)$. Für Papaverin werden diese Veränderungen im Durchschnitt mit 5,4\% (2,9-30\%) angegeben $(4,26)$. Für $\mathrm{PGE}_{1}$ sind derartige Veränderungen extrem selten (13), eine Abhängigkeit von Injektionszahl und -dauer wird diskutiert (4).

\section{Einwilligung}

Die Aufklärung und Einwilligung des Patienten kann unter Verwendung eines Formblattes erfolgen. Trotzdem muß sich der Arzt in einem persönlichen Gespräch davon überzeugen, daß der Patient die Aufklärung verstanden und keine weiteren Fragen mehr hat. Gerade letzteres sollte sich der Therapeut handschriftlich bestätigen lassen, da im Streitfall die Behauptung ungenügender Aufklärung eine Beweislastumkehr zuungunsten des Arztes bewirkt. Bisher sind Schadensfälle bei SKAT selten (18). Mit zunehmender Verbreitung der Therapie werden sie jedoch zunehmen.

Liegt die Einwilligung des gesetzlichen Vertreters vor und sind auch alle anderen eben genannten Voraussetzungen erfültt, sehen wir keine rechtlichen Probleme bei der Behandlung nicht voll geschäftsfähiger Patienten. Aus medizinisch-ethischer Sicht würden wir eine solche Behandlung allerdings nicht durchführen.

\section{Literatur}

${ }^{1}$ Brindley, G. S.: Cavernosal alpha-blockade: a new technique for investigating and treating erectile impotence. Br. J. Psychiat. 143 (1983) $332-337$

2 Deutsch, E.: Anmerkung zum Urteil des OLG Köln vom 30.5.90 Az 27 U 169/89, VersR. 5 (1991) 189

${ }^{3}$ Deutsch, E.: Arzneimittelhaftung. In Deutsch, E. (Hrsg.): Arztrecht und Arzneimittelrecht, Kpt. XXIX., 2. Aufl., Springer Verlag, Berlin, Heidelberg, New York (1991) 417-437

4 Jünemann, K. P., P. Alken: Pharmacotherapy of erectile dysfunction: a review. Int. J. Impotence Res. 1 (1989) 71-93

5 Lakin, M. M., D. K. Montague, S. V. Medendorn, L. Tesar, L. R. Schover: Intracavernous injection therapy: analysis of results and complications. J. Urol. 143 (1990) 1138-1141

${ }^{6}$ Laufs, A.: Heilversuch und klinisches Experiment. In Laufs, $A$. (Hrsg.): Arztrecht, Kpt. XI., 4. Aufl. C. H. Beck Verlag, München (1988) 219-234

7 Levine, S. B., S. E. Althof, L. A. Turner, C. B. Risen, D. R. Bodner, E. D. Kursh, M. I. Resnick: Side effects of selfadministration of intracavernous papaverine and phentolamine for the treatment of impotence. J. Urol. 141 (1989) 54-57
${ }^{8}$ Lue, T. F, J. G. Wayne, J. Hellstrom, W. McAnnich, E. A. Tanagho: Priapism: a refined approach to diagnosis and treatment. J. Urol. 136 (1986) 104-108

${ }^{9}$ Lue, T. E.: Impotence: a patient's goal-directed approach to treatment. World J. Urol. 8 (1990) 67-74

10 OLG Köln, Urteil vom 30.5.90 Az 27 U 169/89, VersR. 5 (1991) 186-189

11 Padma-Nathan, H., I. Goldstein, T. Payton, R. J. Krane: Intracavernosal pharmacotherapy: the pharmacologic erection program. World J. Urol. 5 (1987) 160-165

12 Porst, H., H. van Ahlen: Pharmako-induzierte Priapismen Ein Erfahrungsbericht über 101 Fälle. Urologe A 28 (1989) 84 $-87$

13 Porst, H., H. van Ahlen, T. H. Bloch, W. Halbig, R. Hautmann, D. Löchner-Ernst, J. Rudnick, G. Staehler, H. M. Weber, W. Weidner, W. H. Weiske: Intercavernous self-injection of prostaglandin $E_{1}$ in the therapy of erectile dysfunction. Vasa Suppl. 28 (1989) 50-56

14 Roy, J. B., R. L. Petrone, S. I. Said: A clinical trial of intracavernous Vasoactive Intestinal Polypeptide to induce penile erection. J. Urol. 143 (1990) $302-304$

15 Sander, Köhler: Arzneimittelrecht, Erläuterungen zu \$21 AMG W. Kohlhammer Verlag, Köln (1987)

16 Schwarzer, J. U., R. Hofmann: Purulent corporeal cavernositis secondary to papaverine-induced priapism. J. Urol. 146 (1991) $845-846$

17 Sidi, A. A., K. K. Chen: Clinical experience with vasoactive intracavernous pharmacotherapy for the treatment of impotence. World J. Urol. 5 (1987) 156-159

18 Sparwasser, H. H.: Übersicht über die Auswertung von Gerichts-, Schlichtungskammer- und Versicherungsgutachten, aufgegliedert in typische Risiken. Vortrag Südwestdeutsche Gesellschaft für Urologie, Koblenz 9.-11.5.91 (Abstraktband)

19 Stackl, W. R. Hasum, M. Marberger: The use of prostaglandin $\mathrm{E}_{1}$ for diagnosis and treatment of erectile dysfunction. World J. Urol. 8 (1990) 84-86

20 Stief, C. G., W. F. Thon, U. Wetterauer, F. Schaebsdan, U. Jonas: Calcitonin-Gene Related Peptide (CGRP) - A possible neurotransmitter for human penile erection and its therapeutical application in impotent patients. Int. J. Impotence Res. 2, Suppl. 2 (1990) 22-23

21 Stief, C. G., F. Holmquist, E. P. Allhoff, K. E. Anderson, U. Jonas: Preliminary report on the effect of nitric oxide donor SIN-1 on human cavernous tissue in vivo. World J. Urol. 9 (1991) 237-239

22 Thurk: Recht im Gesundheitswesen. Loseblattsammlung (Stand März 1989). Gesetz über den Verkehr mit Arzneimitteln vom 24. August 1976 in der Fassung vom 20. Juli 1988

23 Virag, R.: Intracavernous injection of papaverine for erectile failure. Lancet 2 (1982) 938

24 Virag, R., K. Shoukry, J. Floresco, F. Nollet, E. Greco: Intracavernous self-injection of vasoaktive drugs in the treatment of impotence: 8-year experience with 615 cases. J. Urol. 145 (1991) $287-293$

25 Wespes, E., C. C. Schulman: Systemic complications of intracavernous papaverin injections in patients with venous leakage. Urology 31 (1988) 114-115

26 Wetterauer, U.: Intracavernous pharmacotherapy for erectile dysfunction. In Jonas, Thon, Stief (Hrsg.): Erectile Dysfunction, 1. Aufl. Springer, Berlin, Heidelberg, New York (1991) $221-236$

Dr. med. I. Schroeder-Printzen

Klinik und Poliklinik für Urologie

der Universität Göttingen

Robert-Koch-Straße 40

3400 Göttingen 